An experimental and theoretical investigation of particlewall impacts in a T-junction

by

D. Vigolo

M. Griffiths

S. RadI

H. A. Stone 



\title{
An experimental and theoretical investigation of particle-wall impacts in a T-junction
}

\author{
D. V I G O L O ${ }^{1}$, I. M. G R I F F I T H S $\mathrm{S}^{2} \dagger$, S. R A D L \\ AND H. A. STON E \\ ${ }^{1}$ Department of Mechanical and Aerospace Engineering, Princeton University, Princeton, NJ \\ 08544 (USA) \\ ${ }^{2}$ Mathematical Institute, 24-29 St Giles', University of Oxford, Oxford, OX1 3LB, England \\ (UK) \\ ${ }^{3}$ Institute for Process and Particle Engineering, Graz University of Technology, Inffeldgasse \\ 21a/II, 8010 Graz, Austria
}

(Received )

Understanding the behaviour of particles entrained in a fluid flow upon changes in flow direction is crucial in problems where particle inertia is important, such as the erosion process in pipe bends. We present results on the impact of particles in a T-shaped channel in the laminar-turbulent transitional regime. The impacting event for a given system is described in terms of the Reynolds number and the particle Stokes number. Experimental results for the impact are compared with the trajectories predicted by theoretical particletracing models for a range of configurations to determine the role of the viscous boundary layer in retarding the particles and reducing the rate of collision with the substrate. In particular a 2D model based on a stagnation point flow is used together with 3D numerical simulations. We show how the simple 2D model provides a tractable way of understanding the general collision behaviour, while more advanced 3D simulation can be helpful in understanding the details of the flow.

\section{Introduction}

When particles are dispersed in a fluid flowing in a channel, and the direction of motion changes abruptly, particle collisions with the constraining surface may occur. For instance, if we consider a pipe flow through an elbow or T-shaped section, where particles are dispersed in the fluid, particle inertia can play a role in leading to an impact that can potentially produce damage of the surface. Such solid particle erosion (SPE) is a well-known phenomenon in industrial pipe systems (see, for example Levy 1995). A wide range of experimental and computational studies have been performed on pipe systems including both straight sections and bends to evaluate the damage caused by SPE. One such technique utilizes jet impingement on a surface in which, for example, the influence on the strike angle or substrate and erodent material may be considered. An alternative approach to study erosion at high impact speeds is to perform a "pot erosion test', in which a rotor spins in a pot filled with the slurry containing the erodent under investigation. A more detailed account of SPE work comprising experimental and computational approaches is presented in table 1.

$\dagger$ The first two authors contributed equally to this work. 
TABLE 1. Summary of significant work in the solid particle erosion (SPE) literature. ${ }^{a}$ refers to typical flow or particle velocity; ${ }^{b}$ refers to experimental (E) or computational (C) work.

\begin{tabular}{|c|c|c|c|c|c|c|}
\hline Reference & Set-up & Erosion Rate Evaluation & $\begin{array}{l}\text { Particle } \\
\text { Size }(\mu \mathbf{m})\end{array}$ & $\begin{array}{l}\text { Particle } \\
\text { Material }\end{array}$ & $\begin{array}{l}\text { Typical } \\
\text { Speed }^{a} \\
(\mathrm{~m} / \mathrm{s})\end{array}$ & $\begin{array}{l}\text { Type } \\
(\mathbf{E} / \mathrm{C})^{b}\end{array}$ \\
\hline Blanchard et al. (1984) & Elbows & $\begin{array}{l}\text { Visual inspection, alternate } \\
\text { layer of } \mathrm{Cu} \text { and } \mathrm{Ag}\end{array}$ & $95-605$ & Sand & 5 & $\mathrm{E}$ \\
\hline Benchaita et al. (1983) & $\begin{array}{l}\text { Jet impingement }\left(90^{\circ}\right) \text { on metallic } \\
\text { plate }\end{array}$ & $\begin{array}{l}\text { Surface profile by a 'pick up' } \\
\text { needle system }\end{array}$ & $200-1000$ & Sand & $5.5-8$ & $\mathrm{E} / \mathrm{C}$ \\
\hline Shivamurthy et al. (2010) & $\begin{array}{l}\text { Jet impingement }\left(30^{\circ}, 45^{\circ}, 60^{\circ} \text {, }\right. \\
\left.90^{\circ}\right) \text { on Stellite and Colmonoy }\end{array}$ & $\begin{array}{l}\text { SEM images and weight loss } \\
\text { measurement }\end{array}$ & 100,375 & $\mathrm{SiO}_{2}$ & 12 & $\mathrm{E}$ \\
\hline Zhao et al. (1995) & Jet impingement $\left(90^{\circ}\right)$ on steel & $\begin{array}{l}\text { Surface profile and weight loss } \\
\text { measurement }\end{array}$ & $105,149,210$ & $\mathrm{SiO}_{2}$ & 1.7 & $\mathrm{E}$ \\
\hline Zhang et al. (2007) & Jet impingement $\left(90^{\circ}\right)$ & Electrical resistance $(\mathrm{ER})$ probe & 25,150 & Sand & $2.5,5,10$ & $\mathrm{E} / \mathrm{C}$ \\
\hline Lynn et al. (1991) & $\begin{array}{l}\text { Erosion pot tester, particle in oil; } \\
\text { evaluation of impact energy }\end{array}$ & SEM images & $20-500$ & $\mathrm{SiC}$ & 18.7 & $\mathrm{E}^{\prime}$ \\
\hline Clark (1991) & $\begin{array}{l}\text { Erosion pot tester, water and wa- } \\
\text { ter/glycerin mixture; viscosity de- } \\
\text { pendence }\end{array}$ & SEM images & $75-750$ & Glass & $9.35,18.7$ & $\mathrm{E}$ \\
\hline Wood \& Jones (2003) & $\begin{array}{l}\text { Bends, straight section and jet im- } \\
\text { pingement }\left(90^{\circ}\right)\end{array}$ & $\begin{array}{l}\text { Weight loss, profilometer and } \\
\text { Electrical resistance tomogra- } \\
\text { phy (ERT) measurements }\end{array}$ & $500-2000$ & Sand & $\begin{array}{l}0.5, \quad 1, \\
1.5,2,2.5 \\
\text { Jet: } 3.5\end{array}$ & $\mathrm{E}$ \\
\hline Levy et al. (1987) & $\begin{array}{l}\text { Jet impingement, slurry on steel; } \\
\text { strike angle dependence }\end{array}$ & SEM images & 44,79 & $\begin{array}{l}\mathrm{SiO}_{2}, \text { coal, } \\
\mathrm{SiC}\end{array}$ & $10,20,30$ & $\mathrm{E}$ \\
\hline Chen et al. (2006) & $\begin{array}{l}\text { Plugged T-shaped channel and } 90^{\circ} \\
\text { elbow }\end{array}$ & $\begin{array}{l}\text { Aluminium specimens checked } \\
\text { by profilometer }\end{array}$ & 150 & Sand & 3 & $\mathrm{E} / \mathrm{C}$ \\
\hline McLaury et al. (1997) & Elbows and straight pipe & - & 300 & Sand & 6 & $\mathrm{C}$ \\
\hline
\end{tabular}


In all of the indicated literature the principal goal is to evaluate the damage to the boundaries, and hence the results depend strongly on the chosen material and/or on the erosion model applied. In this paper we approach this problem in a more general way, by focusing experimentally and theoretically on the specific particle path towards the wall that leads to an impact, rather than the actual damage caused. In particular, we consider high-Reynolds-number laminar, particle-laden flows within a T-junction, which are characterized by the particle size and density, the details of the flow field, and the system geometry. We evaluate particle trajectories by direct visualization of a range of experiments, and from their traces collect information on local direction and speed, final strike angle and position.

There is a wealth of literature describing the motion of particles within similar geometries for a variety of Reynolds numbers. However, the majority of this work focuses on much smaller devices, often with different arrangements of inlets and outlets. For example, in the extensive work of Kockmann and coworkers (Haller et al. 2009; Kockmann \& Roberge 2011) the flow in a microfluidic L-bend, T-channel or fork-shaped element is considered. In particular, for the case of a T-junction the authors characterize the flow and the vortex formation at the junction in the range $0<R e<700$, focusing on the heat-transfer properties and pressure drop.

Many papers consider the flow mixing that arises in small T-shaped junctions where two miscible liquids enter the lateral branches of a T-junction, meeting and eventually mixing as they flow together in the converging channel towards the outlet. For example, experiments and simulations are presented in Wong et al. (2004), where a performance test on the mixing capabilities of such a configuration is tested, and in Adeosun \& Lawal (2009), an evaluation of the flow and the mixing properties based on residence time distribution is shown. In Dreher et al. (2009) flows up to $R e=1000$ are studied numerically in more detail, showing a periodicity in the mixing quality that reach a maximum at about $R e=500$. Another flow configuration often used to study mixing phenomena is that in which the two inlets meet perpendicularly to each other at the T-junction and then move towards the outlet. Such a configuration has been studied, for example, by Bruecker (1997) using three-dimensional particle image velocimetry. Here, analogies of the vortical structure with a jet in a cross flow and their implication in mixing performance are discussed. Although the geometry is essentially the same as the conventional T-junction we consider in this paper, the flow configuration is completely different for mixing applications, and no particle-wall impact is considered.

In Li et al. (2012) the collisions of particles immersed in a liquid with a solid surface are examined and the restitution coefficient (defined as the ratio between the rebound to the impact velocity) is found for fairly large and heavy metal spheres (of diameter $9.5 \mathrm{~mm}$ ), dropped in steady water from different heights to vary the particle Reynolds number. The results of these experiments are compared with numerical simulations. In Yang \& Hunt (2008) a mixed contact model is discussed. Here a single particle approaching a solid surface while immersed in a liquid is analysed and a model is developed for the impact, that considers the effect of both the asperity of the surface and the presence of a liquid in the interstitial region between the approaching particle and the wall. In both of these works, however, no external flow is present, in contrast with the work we present here for a particle-laden flow.

The influence of wall roughness on impact behaviour is experimentally investigated in Sommerfeld \& Huber (1999) where a particle dispersion in air is made to flow in a horizontal channel. Particle collisions with side walls and the resulting rebounds are studied for various impacting surfaces and particle sphericity. Experimental results are then used to improve a collision model and its predictions. Other studies on particle-wall 
collisions are also performed by Tu and coworkers (Tu 2000; Tu et al. 2004), in which the high-speed impact of a gas-particle flow on a cylindrical body is studied in the range $8000<R e<100000$. Experiments and simulations on impact and rebounding particles reveal that the particle behaviour and rebounding flow pattern near the wall is mainly dominated by particle inertia.

The aim of our work here is to consider a comprehensive scenario in which a dispersion of particles is made to flow in a high-Reynolds-number laminar flow while abruptly changing direction at a T-junction. The majority of the literature related to the study of similar flow regimes focuses attention on an inviscid flow (see for example Benchaita et al. (1983)). In this paper we consider a spectrum of flow configurations in larger Tjunction configurations, that allow for the size of the viscous boundary layer, adjacent to the wall, to be non-negligible when compared with the size of a particle. Consequently, the viscous boundary layer may have an appreciable effect on the particle behaviour and impact dynamics. In addition we obtain insight into the key parameters that govern whether a particular configuration will generate wall impacts by considering a fully three-dimensional simulation of the flow behaviour. We exploit observations made about the flow field to derive a reduced two-dimensional model that is used to show that the system behaviour is captured via three key dimensionless parameters, which describe respectively the flow field, the entrained-particle characteristics, and the system geometry. The two-dimensional model is validated against both the three-dimensional simulations and the experimental observations. Finally, the theory is used to present phase diagrams that capture the features of any laminar flow field, viscous boundary layer and particle character to determine whether a system configuration will give rise to particle impacts.

\section{Experimental set-up}

To study the influence of inertia on the particle-wall collisions, an aqueous dispersion of particles of different sizes and materials are injected into a flow in a transparent polycarbonate T-shaped channel with a square section of lateral dimensions $L=0.48 \mathrm{~cm}$. In particular, we vary the particle-to-fluid density ratio in the range $1-4.5$ by choosing beads made of Polymethyl methacrylate (PMMA, with particle density $\rho_{p}=1180 \mathrm{~kg} / \mathrm{m}^{3}$ ), ordinary silica glass $\left(\mathrm{SiO}_{2}, \rho_{p}=2500 \mathrm{~kg} / \mathrm{m}^{3}\right), \mathrm{ZrO}_{2} / \mathrm{SiO}_{2}$ glass $\left(\rho_{p}=3800 \mathrm{~kg} / \mathrm{m}^{3}\right)$, and high density barium titanate (TiBa) glass $\left(\rho_{p}=4500 \mathrm{~kg} / \mathrm{m}^{3}\right)$. In addition, we cover more than an order of magnitude in particle radii ranging over $a=10-350 \mu \mathrm{m}$ (see table 2). A sketch of the set-up used in the experiments is provided in figure 1(a). The fluid is forced to flow through the channel by a centrifugal pump (ViaAqua 3300), which enters the device from the top and exits laterally via two outlets. Because of the symmetry, the flow rate at one of the outlets is exactly half of the flow rate at the inlet.

The fluid used is a mixture of deionized water and glycerol (Sigma-Aldrich) in order to vary the viscosity $(\mu)$ and density $\left(\rho_{f}\right)$ of the fluid to cover a wide range of Reynolds numbers, $R e$, defined as

$$
R e=\frac{\rho_{f} U L}{\mu},
$$

where $U$ is the volume-averaged fluid velocity at the inlet. The glycerol-water ratios used, the respective densities and viscosities of the fluids, the average fluid speed that could be attained at the highest flow rate, and the associated channel Reynolds numbers are shown in table 3.

A typical experiment is conducted by recording high-speed images (up to 35,000 fps) of the impacting particles using a high-speed camera (Vision Research, Phantom v7.3) and 

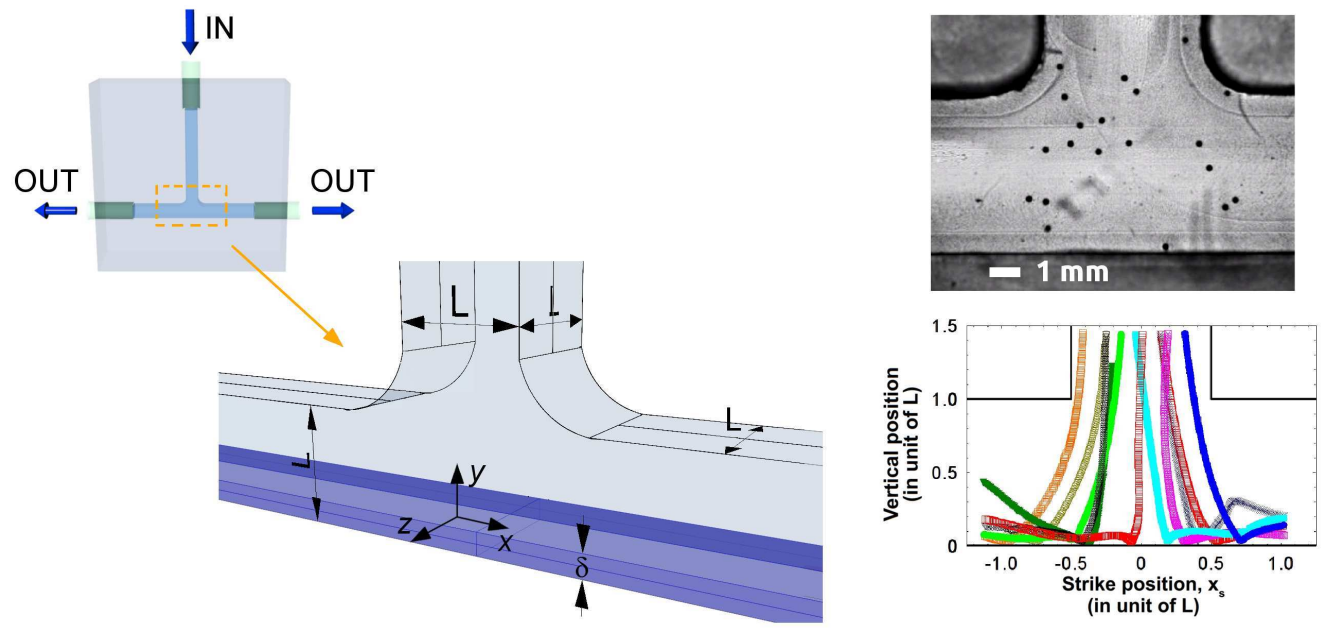

FIGURE 1. A sketch of the T-shaped channel used in the experiments with the lateral size of the square-section channel, $L$, and the viscous boundary-layer thickness, $\delta$. (b) A high-speed image of the impact zone where pure water is seeded with $97 \mu$ m-radius TiBa glass particles. (c) A sequence of high-speed images of particle positions that depict the trajectories of the impacting particles.

TABLE 2. Characteristics of the particles used in the experiments. The particles were supplied by ${ }^{a}$ MO-SCI Specialty Products L.L.C.; ${ }^{b}$ GlenMills Inc.; ${ }^{c}$ Microbeads Spheromers CA 20. ${ }^{\ddagger}$ Particle size tested but not shown in the experimental results below.

\begin{tabular}{lll} 
Material & $\boldsymbol{\rho}_{\boldsymbol{p}}\left(\mathbf{k g} / \mathbf{m}^{\mathbf{3}}\right)$ & $\boldsymbol{a}(\boldsymbol{\mu} \mathbf{m})$ \\
\hline $\mathrm{Ti} \mathrm{Ba} \mathrm{glass}^{a}$ & 4500 & $21,50^{\ddagger}, 69^{\ddagger}, 97,163^{\ddagger}$ \\
$\mathrm{ZrO}_{2} / \mathrm{SiO}_{2}{ }^{b}$ & 3800 & 350 \\
$\mathrm{SiO}_{2}{ }^{b}$ & 2500 & $75,128,175$ \\
$\mathrm{PMMA}^{c}$ & 1180 & 10
\end{tabular}

TABLE 3. Characteristics of the fluid used in the experiments. ${ }^{\S}$ Average velocity corresponding to the highest flow rate achievable under these conditions.

\begin{tabular}{|c|c|c|c|c|}
\hline Glycerol (\%) & $\boldsymbol{\rho}_{\boldsymbol{f}}\left(\mathrm{kg} / \mathrm{m}^{3}\right)$ & $\boldsymbol{\mu}(\mathrm{Pas})$ & $J^{\S}(\mathrm{m} / \mathrm{s})$ & $R e$ \\
\hline 0 & 997 & 0.00089 & 1.21 & 6500 \\
\hline 10 & 1021 & 0.00114 & 1.17 & 5000 \\
\hline 20 & 1045 & 0.0015 & 1.09 & 3600 \\
\hline 30 & 1071 & 0.0021 & 1.06 & 2600 \\
\hline 40 & 1097 & 0.0032 & 1.01 & 1700 \\
\hline 50 & 1124 & 0.005 & 0.91 & 980 \\
\hline 60 & 1151 & 0.0087 & 0.76 & 480 \\
\hline 70 & 1178 & 0.017 & 0.47 & 160 \\
\hline
\end{tabular}

by tracing the particle trajectories using an in-house MATLAB code. An example of the results obtained from the image analysis is given in figures 1(b) and (c), where we show the measured trajectories of an ensemble of particles impacting the bottom surface of the T-shaped channel. The particles may be traced by performing the experiments with 
the camera focused in the plane $z=0$, i.e., at the centre of the channel. Our MATLAB routine is designed to track only particles that are perfectly in the focal plane and, as we are using an objective with a wide aperture (i.e., a Sigma 105mm F2.8 EX DG Macro), the majority of the measured trajectories are based on particles that are exactly in the $z=0$ plane.

Our particle-tracing approach provides two-dimensional information on the instantaneous position, speed and direction of each particle, and, in the case of a collision, it is possible to obtain information on the strike speed and angle. While we cannot measure the third (i.e., depth) component of the particle velocity (and hence the true strike angle), we argue that this velocity component is of relatively little importance, since the velocity components in the main flow direction are much larger than that in the spanwise direction. This feature is especially true when looking at the mid-plane of the T-junction, as shown in the next section.

A key parameter in determining the influence of hydrodynamic effects on whether a particle will impact a surface is the thickness of the viscous boundary layer on the lower boundary, which is given by $\delta=\sqrt{\mu / \rho_{f} \dot{\gamma}}$ where $\dot{\gamma}=U / L$ is the typical shear rate in the T-junction channel. Consequently, $\delta=L / \sqrt{R e}$. Also, we define $\epsilon=a / L$ as the ratio of the radius of an entrained particle, $a$, to the lateral size of the T-junction, $L$. In the experiments conducted here, $\epsilon$ is small and so the influence of the particle motion on the fluid flow is negligible. However, the ratio of the particle size to the boundary layer thickness, $\alpha$ is given by

$$
\alpha=\frac{a}{\delta}=\epsilon \sqrt{R e},
$$

which, since the Reynolds number can be large, is not necessarily small. In the experiments conducted here $\alpha$ ranges between $10^{-2}$ and 10, and thus the viscous boundary layer can have an appreciable effect on the particle trajectories.

\section{Modelling strategy to derive the flow field}

By performing direct numerical simulations of the Navier-Stokes equations, we are able to obtain a detailed three-dimensional description of the flow field as a function of the Reynolds number. Since we are unable to accurately measure experimentally the particle motion in the direction of the channel's depth, this approach provides a better understanding, for the detailed flow field and particle trajectories. In addition, we exploit observations from the simulations that allow us to develop a simplified two-dimensional model.

We study a dimensionless system, whereby lengths are non-dimensionalized with the lateral pipe size, $L$, velocities with the average fluid velocity, $U$, and time with $L / U$ :

$$
\boldsymbol{x}^{*}=L \boldsymbol{x}, \quad \boldsymbol{u}^{*}=U \boldsymbol{u}, \quad t^{*}=\frac{L}{U} t .
$$

In the following subsections, we present the three-dimensional modelling approach and the reduced two-dimensional model used to describe the flow field.

\subsection{Three-dimensional modelling}

Firstly, three-dimensional simulations were performed using OpenFOAM, an open-source, transient numerical algorithm to solve the Navier-Stokes equations for an incompressible fluid flow. A suitably fine computational grid (1.4 Mio. cells) was employed to ensure appropriate resolution of the boundary layer near the impacting region of the incoming fluid. The details of this numerical approach are summarized in Appendix A. 


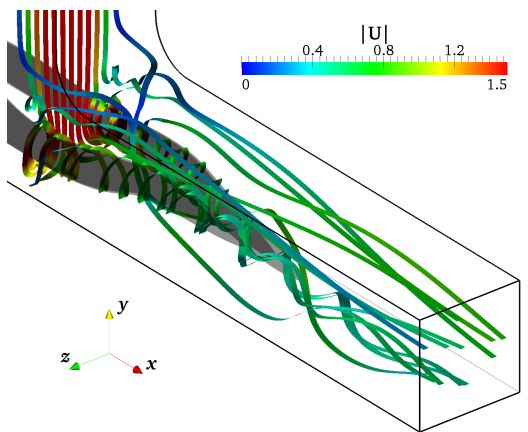

(a)

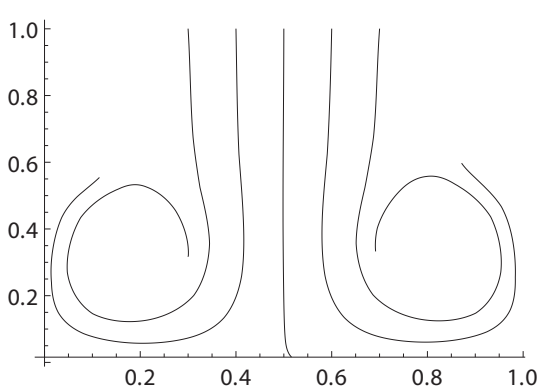

(b)

FIgURE 2. (a) Three-dimensional streamlines (coloured by the magnitude of the fluid velocity) and iso-contours of the pressure field (shaded regions) based on a direct numerical simulation of the flow in a T-junction $(R e=500)$; (b) Streamlines in the $y z$-plane of a T-junction.

Typical streamlines based on the results of the numerical simulations are sketched in figure 2(a), which illustrate the complex (laminar) flow features that develop at even moderately large Reynolds numbers. The swirl of the flow in the form of two counter-rotating vortices increases gradually with increasing Reynolds number, and the flow becomes more complex, with two large vortices arising near the impingement region and two smaller vortices above them (see figure 2(a)). The highest streamwise velocity is located at approximately $x=0.60$, and this high velocity region moves closer to the wall (and hence the characteristic boundary layer thickness decreases) with increasing Re. For Reynolds numbers $R e \lesssim 540$, fluid parcels initially in the plane $z=0$ remain in the plane, i.e., the $z$-component of the fluid velocity is zero, and the flow is approximately symmetric about the $y z$-plane, e.g., see figure 2(b). Furthermore, the streamlines in the three-dimensional simulations suggest that those particles that lie in the plane $z=0$ are the most likely to impact the lower surface, while particles out of this plane will become entrained in vortices. These features motivate our simplified model in the next section.

The flow pattern in the plane $z=0$ is shown for various Reynolds numbers in figure 3 . At low Reynolds numbers, for example $R e=10$ (figure 3(a)), streamlines are parallel, and the velocity profile in the two outlet channels is nearly symmetric. For Reynolds numbers up to $R e=540$, we observe a steady flow (see results for $R e=100$ and $R e=500$ in figures $3(b)$ and $(c)$ ). In figures $3(d)$ and (e) typical results for an unsteady flow behaviour are shown. Clearly, streamlines starting at $z>0$ are then able to cross the $x y$-mid-plane of the channel, and velocity fluctuations are generally $O(1)$.

Despite the many complex flow features uncovered by the Navier-Stokes solver, it is clear from the simulations (figure 2(b)) that considering particles in the plane $z=0$ will capture the most significant dynamical information on particles that are most likely to impact the lower wall of the T-junction, up to moderately high Reynolds numbers. These simulations motivate us to examine a reduced, tractable two-dimensional formulation of the system that is more amenable to parameter analysis. In the following subsection we establish a simpler two-dimensional model, where we also neglect the presence of the lateral walls. Our model will allow us to obtain a limit curve that separates the parameter regime where particle-wall collisions occur from that where no collisions are observed. 


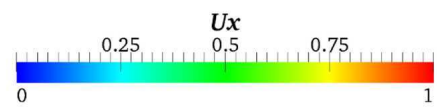

(a)
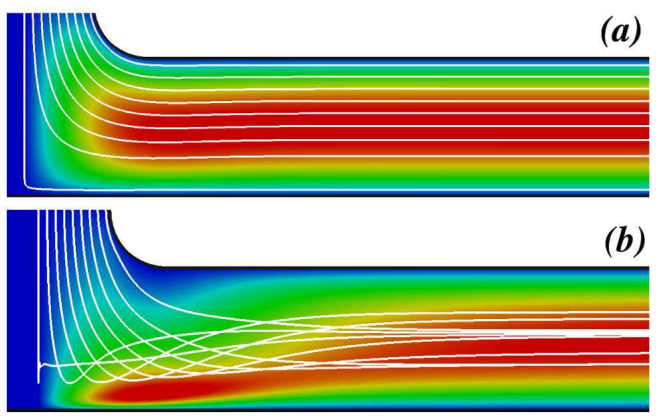

(b)

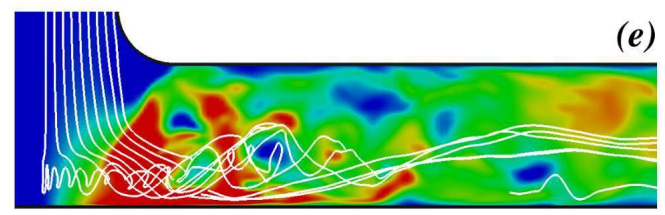

Figure 3. Contour plot of the instantaneous streamwise (i.e., $x$ ) velocity component at the centre of the channel $(z=0)$, as well as streamlines starting at $z=0.3$ for various Reynolds numbers (streamlines in panel $(d)$ and (e) piercing the plane $z=0$ are not shown). (a) $R e=10$, (b) $R e=100$, (c) $R e=500$, (d) $R e=600$, (e) $R e=2000$.

\subsection{Two-dimensional modelling}

In reducing the dimensionality of the system to focus on the particles in the $z=0$ plane, which are most likely to impact on the channel wall, we may approximate the T-junction flow as a two-dimensional extensional flow. In this model, we assume that the flow field near the lower boundary may be approximated by a stagnation-point flow, as studied for example by Benchaita et al. (1983). However, unlike in this work where the size of the viscous boundary layer is assumed to be small in comparison to the particle size, here we cannot neglect the effect of the viscous boundary layer. We therefore consider a model that also incorporates this effect and investigate the implications and results of this flow feature on the predicted particle behaviour. However, as discussed in $\S 2$, since the particles are much smaller than the channel geometry (i.e., $\epsilon \ll 1$ ) we assume they will not influence the fluid flow.

We assume that particles enter in the mid-plane $z=0$ at position $\boldsymbol{x}_{0}=\left(x_{0}, y_{0}\right)$ in Cartesian coordinates (see figure 1 for the coordinate geometry) into a viscous twodimensional stagnation-point flow, which is given by the Hiemenz solution (Hiemenz 1911),

$$
u=x f^{\prime}(\sqrt{R e} y), \quad v=-\frac{1}{\sqrt{R e}} f(\sqrt{R e} y),
$$

where $f$ satisfies (primes denote derivatives)

$$
\begin{gathered}
f^{\prime \prime \prime}+f f^{\prime \prime}-\left(f^{\prime}\right)^{2}+1=0, \\
f(0)=f^{\prime}(0)=0, \quad f^{\prime} \rightarrow 1 \quad \text { as } y \rightarrow \infty .
\end{gathered}
$$

The Reynolds number represents a measure of the relative thickness of the viscous boundary layer, which scales with $1 / \sqrt{R e}$ as displayed in figure 4 . As $y \rightarrow \infty, u \rightarrow x$ and $v \rightarrow-y$ which corresponds to the solution for an inviscid stagnation-point flow. We assume that the particles enter with velocity $\boldsymbol{u}_{0}=\left(0, v_{0}\left(x_{0}, y_{0}\right)\right)$, where $v_{0}<0$. The choice of the parameter $y_{0}$ corresponds to the entry point for the particles and here we choose $y_{0}=1$ so that the effect of the stagnation-point flow is observed at a distance of one pipe cross- 


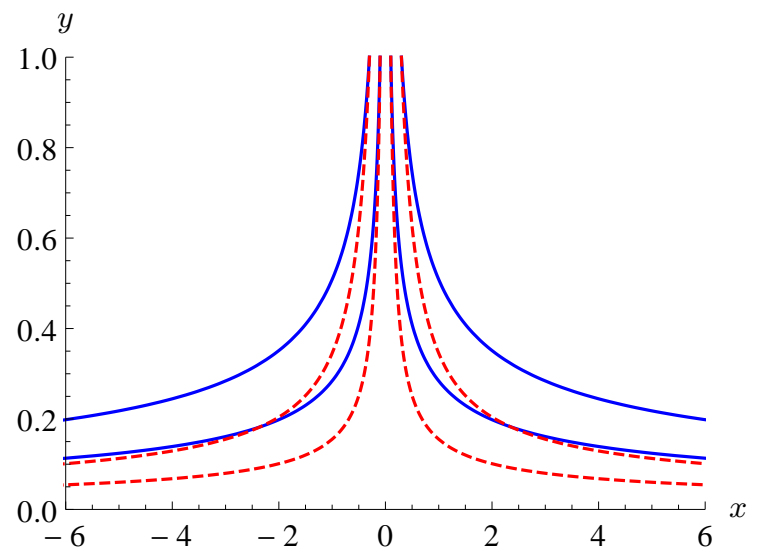

Figure 4. Streamlines for the Hiemenz viscous stagnation-point flow, equation (3.2), for $R e=1$ (blue) and $R e=100$ (red dashed), which illustrates the effect of the viscous boundary layer.

sectional length from the impacting wall (this value is also taken by Benchaita et al. (1983)). We note that the Hiemenz solution equation (3.2) for the flow field may be generalized to three dimensions by the Homann flow solution (Homann 1936), but here we are concerned only with the two-dimensional solution, which captures the flow behaviour relevant to our analysis of impact.

\section{Particle trajectories}

There are various forces that contribute to the motion of the particles suspended in a flowing fluid. Whilst the viscous drag force will tend to guide particles along streamlines, other forces, for example those arising as a result of particle inertia and lift, will cause the particles to deviate from the streamlines. As a result, appropriate force models must be chosen since we cannot resolve the details of the flow around individual particles. There are several potentially relevant forces that we detail below.

The principal force exerted on a particle is the drag due to the viscous resistance of the surrounding fluid. Specifically, for a given flow field the particles experience a dimensional drag force, $\boldsymbol{F}_{D}^{*}$, which we assume to be (approximately) quadratic in nature, as in Benchaita et al. (1983),

$$
\boldsymbol{F}_{D}^{*}=\frac{C_{D}}{2} \rho_{f} \pi a^{2}\left(\boldsymbol{u}^{*}-\dot{\boldsymbol{x}}_{p}^{*}\right)\left|\boldsymbol{u}^{*}-\dot{\boldsymbol{x}}_{p}^{*}\right|,
$$

where $\boldsymbol{x}_{p}^{*}$ denotes the dimensional particle position, dots denote time derivatives, and $C_{D}$ is the drag coefficient. For example, the Khan-Richardson model expresses the force on a particle for a large dynamic range of Reynolds numbers, $10^{-2}<R e<10^{5}$ (Richardson et al. 2002) and has the form

$$
C_{D}=2\left(1.849 R e_{p}^{-0.31}+0.293 R e_{p}^{0.06}\right)^{3.45},
$$

where $R e_{p}$ is the particle Reynolds number, defined by

$$
R e_{p}=\frac{\rho_{f}\left(U-U_{p}\right) L}{\mu} .
$$

Here $U$ and $U_{p}$ represent typical fluid and particle speeds. We may use this definition 
to allow us to write the particle Reynolds number in terms of the Reynolds number and the dimensionless particle flow speed via

$$
R e_{p}=R e\left|\boldsymbol{u}-\dot{\boldsymbol{x}}_{p}\right| .
$$

The dependence of the drag coefficient on the local particle and fluid velocity means that we first solve for the flow and then determine the drag coefficient, $C_{D}$, using equation (4.2) a posteriori.

Other potentially relevant forces experienced by the particles within the bulk fluid flow are (* denotes dimensional variables): the drag due to a mean pressure gradient acting on the surface of the particle,

$$
\boldsymbol{F}_{p}^{*}=-2 \pi a^{3} \nabla^{*} p^{*}
$$

where $p^{*}$ is the dimensional fluid pressure (Clift et al. 1978); added mass forces, which originate from the acceleration of the surrounding fluid (Clift et al. 1978),

$$
\boldsymbol{F}_{a}^{*}=\frac{2}{3} \pi a^{3} \rho_{f} \frac{\mathrm{d}}{\mathrm{d} t^{*}}\left(\boldsymbol{u}^{*}-\dot{\boldsymbol{x}}_{p}^{*}\right) ;
$$

lift due to stresses induced by fluid and/or particle rotation (Rubinow \& Keller 1961),

$$
\boldsymbol{F}_{l}^{*}=C_{L} \rho_{f} \pi a^{3}\left(\boldsymbol{u}^{*}-\dot{\boldsymbol{x}}_{p}^{*}\right) \times \boldsymbol{\omega}^{*},
$$

where $\boldsymbol{\omega}^{*}$ is the angular velocity of the particle and $C_{L}$ is the corresponding lift coefficient; and gravity,

$$
\boldsymbol{F}_{g}^{*}=\frac{4}{3} \pi a^{3}\left(\rho_{p}-\rho_{f}\right) \boldsymbol{g}
$$

where $\boldsymbol{g}$ is the acceleration due to gravity.

As the particles approach the wall, they also experience lubrication forces due to the viscous flow between the particle surface and a surrounding surface, in both the normal and tangential directions to the wall. We model the normal force experienced via the model proposed by (Goldman et al. 1967),

$$
\boldsymbol{F}_{n}^{*}=-6 \pi \mu a^{2} \frac{\dot{y}_{p}^{*}}{\left(y_{p}^{*}-a\right)} \boldsymbol{e}_{\boldsymbol{y}}
$$

where $\boldsymbol{e}_{\boldsymbol{y}}$ denotes the unit vector in the $y^{*}$-direction, perpendicular to the wall. As $y_{p}^{*} \rightarrow a$ and the particle approaches the wall (recalling that $\boldsymbol{x}^{*}$ denotes the coordinate of the centre of the particle so when $y_{p}^{*}=a$ the particle is touching the wall), the normal force diverges, indicating that a particle can never strike a perfectly smooth wall. In practice, however, both the surfaces of the wall and the particle are not perfectly smooth. In our model we therefore classify a strike when the particle comes within a distance $y_{p}^{*}=a+b$, where $b$ is a characteristic roughness of the wall (we assume the particles to be perfectly smooth here). The corresponding dimensionless parameter $\beta=b / L$ characterizes the roughness of the wall. In our experiments, the typical wall roughness of the polycarbonate channel is $5 \mu \mathrm{m}$, which leads to a roughness parameter $\beta=1 \times 10^{-3}$; we use this value of $\beta$ in our simulations.

The tangential force experienced by a particle in the proximity of a wall, $\boldsymbol{F}_{t}^{*}$, is approximated by (Pasol et al. 2011),

$$
\boldsymbol{F}_{t}^{*}=-\frac{16 \pi \mu a}{5} \log \left(\frac{a}{y_{p}^{*}-a}\right) \dot{x}_{p}^{*} \boldsymbol{e}_{\boldsymbol{x}}
$$

when $y^{*}-a \ll a$ and the particle is much closer to the wall than its radius. Here $\boldsymbol{e}_{\boldsymbol{x}}$ 


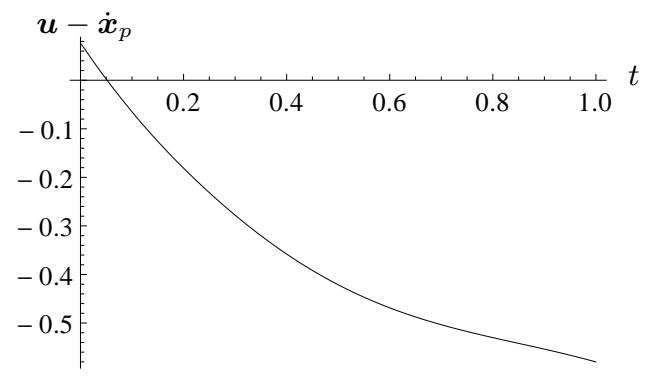

Figure 5. Plot of $\left(\boldsymbol{u}-\dot{\boldsymbol{x}}_{p}\right)$ versus $t$ for a particle with $S t=0.35$ travelling in a flow characterized by $R e=100$ in the presence of a viscous boundary layer with $\alpha=1$.

and $\boldsymbol{e}_{\boldsymbol{z}}$ denote, respectively, unit vectors in the $x^{*}$ and $z^{*}$ directions. To ensure that this force tends to zero as we move away from the wall we use a suitable representation that captures the force close to the wall while matching to the required far-field behaviour, which leads to the uniformly valid tangential force

$$
\boldsymbol{F}_{\mathrm{uni}, t}^{*}=-\frac{16 \pi \mu a}{5} \log \left(1+\frac{a}{y_{p}^{*}-a}\right) \dot{x}_{p}^{*} \boldsymbol{e}_{\boldsymbol{x}} .
$$

The resulting dimensionless equation that governs the particle trajectories is thus given by

$$
\begin{aligned}
& S t \ddot{\boldsymbol{x}}=\frac{C_{D}}{2}\left(\boldsymbol{u}-\dot{\boldsymbol{x}}_{p}\right)\left|\boldsymbol{u}-\dot{\boldsymbol{x}}_{p}\right|-2 \epsilon \boldsymbol{\nabla} p+\frac{2}{3} \epsilon \frac{\mathrm{d}}{\mathrm{d} t}\left(\boldsymbol{u}-\dot{\boldsymbol{x}}_{p}\right)+\epsilon C_{L}\left(\boldsymbol{u}-\dot{\boldsymbol{x}}_{p}\right) \times \omega \\
& +\frac{4}{3} \frac{\epsilon}{F r^{2}}\left(\frac{\rho_{p}}{\rho_{f}}-1\right) \hat{\boldsymbol{g}}-\frac{6}{\sqrt{R e}} \frac{\dot{y}_{p}}{\left(\sqrt{R e} y_{p}-\alpha\right)} \boldsymbol{e}_{\boldsymbol{y}}-\frac{16}{5 \alpha \sqrt{R e}} \log \left(\frac{\sqrt{R e} y_{p}}{\sqrt{R e} y_{p}-\alpha}\right) \dot{x}_{p} \boldsymbol{e}_{\boldsymbol{x}}
\end{aligned}
$$

where $p$ is the dimensionless fluid pressure, scaled with $\rho_{f} U^{2}$, and $\hat{\boldsymbol{g}}$ is a unit vector in the direction of gravity. Here we define

$$
S t=\frac{4}{3} \frac{\rho_{p}}{\rho_{f}} \frac{a}{L}
$$

as a characteristic Stokes number that represents the ratio of particle inertia to the fluid drag force; particles deviate progressively from streamlines with increasing Stokes number. In equation (4.12) the parameter $F r=U / \sqrt{L g}$ is the Froude number and reflects the relative importance of viscous to gravity forces. Since $\epsilon \ll 1$ we neglect the force terms on the right-hand side corresponding to pressure, added mass, lift and gravity so that the important forces on the particle are only the drag force and the lubrication forces experienced by the particle in the proximity of an external wall.

As described above, the force relation (4.12) may be solved for a given flow field to determine the particle Reynolds number, and thus the drag coefficient via (4.2). Typical values of $R e_{p} / R e$ in the regime we consider are in general $-10^{-1} \lesssim R e_{p} / R e \lesssim 1$; figure 5 considers the variation of $\boldsymbol{u}-\dot{\boldsymbol{x}}_{p}$ with time for a sample particle trajectory as it transits the T-junction.

Recalling that the flow profile and drag coefficient depend only on $R e$, and that we have neglected all terms involving $\epsilon$, we find that the trajectories of the particles depend on three independent parameters: $R e, S t$ and $\alpha$, representing respectively the effect of the surrounding flow field, the nature of the particles, and the relationship between particle 
size and the flow conditions, where we recall that $\alpha$ is the ratio of particle size to boundary layer thickness.

The particle trajectories are determined by solving equation (4.12) via an AdamsBashforth multistep method in Mathematica ${ }^{\circledR}$, with the flow conditions set by either the two-dimensional Hiemenz stagnation-point flow model, equation (3.2), or the flow profile obtained from the three-dimensional simulations. In both cases the particle tracing provides information on whether or not a dispersion of particles, characterized by their Stokes number, St, will collide with the bottom surface of a T-junction with wall roughness given by $\beta$, in a channel flow characterized by the Reynolds number, $R e$, and the ratio of the particle size to depth of the viscous boundary layer, $\alpha$.

\section{Model predictions}

Before using the reduced 2D model equations (3.2) and (4.12) to make predictions on the possibility of a collision we first validate the theory. This step is achieved by comparing the predictions on the effect of the viscous boundary layer with the results of two sets of experiments. First, we compare the predicted speed at impact versus the position of impact, and the corresponding experimental results obtained from the high-speed camera data as shown in figure $6(a)$. Both results show a strike velocity that increases monotonically with the strike position: $v_{s} \propto x_{s}^{2}$ for $\left|x_{s}\right| \lesssim R$ and $v_{s} \propto x_{s}$ for $\left|x_{s}\right| \gtrsim R$, and moreover the theoretical and experimental predictions are in good agreement. We emphasize this agreement since the set-up comes with no fitting parameters, with all system parameters being determined explicitly for a given experiment.

Second, we find that the inclusion of the viscous boundary layer has a significant effect on the trajectories of particles that are comparable to or smaller than the thickness of the boundary layer, corresponding to solutions for which $\alpha$ is not too large. In figure $6(b)$ and its inset, we observe an approximately linear dependence of the impact position, $x_{s}$, on the initial position, $x_{0}$, of the particle. Cases are presented for two different values of $\alpha$ and, despite the experimental data being more scattered, good agreement with the theoretical predictions is still evident. In particular, as predicted by the theory, when $\alpha$ decreases the slope of the predicted curve and of the distribution of the experimental data points increases, which means that the presence of the viscous boundary layer changes the particles' trajectories and so results in impact positions more distant from the initial position at the inlet. As above, no fitting parameters were used in the comparison shown in the plot.

Having validated the mathematical model we now turn our attention to the dependence of the system behaviour on the dimensionless parameters that characterize the wallimpact problem. In figure $7(a)$ we present a phase-plane prediction in terms of the Stokes and Reynolds numbers. The results of the $2 \mathrm{D}$ analysis illustrate an idealized divide into two zones: an 'impact zone', where particles will collide with the wall, and a 'non-impact zone', where particles injected into the flow will not touch the surface of the channel perpendicular to the inlet flow. The limit curve is validated by comparison with the more comprehensive 3D simulations, which, for a given simulation, provides a predicted point on this data curve, for $R e=10,100$ (shown by the + symbols). The agreement is excellent and crucially validates the predictions of the simplified $2 \mathrm{D}$ model. It is clear that to determine the limit curve from 3D simulations would require many simulations, being both computationally expensive and time consuming. We thus conclude that the 2D model for particles within the two-dimensional Hiemenz viscous stagnation-point flow, equation (3.2), and the force law equation (4.12), offers a simple way of determining the continuous limit curve that separates the regions of impact and non-impact of the 


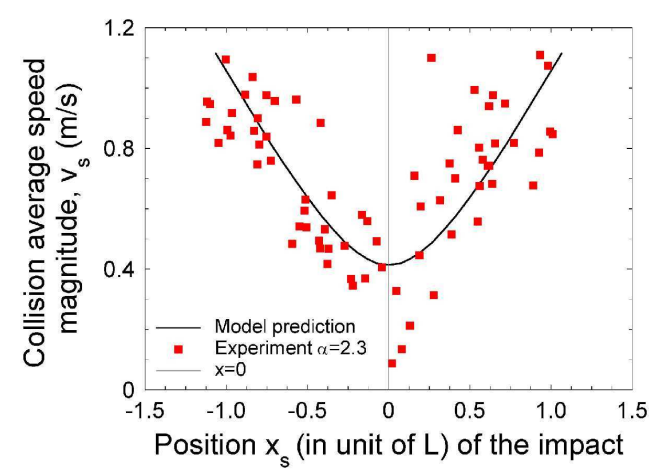

(a)

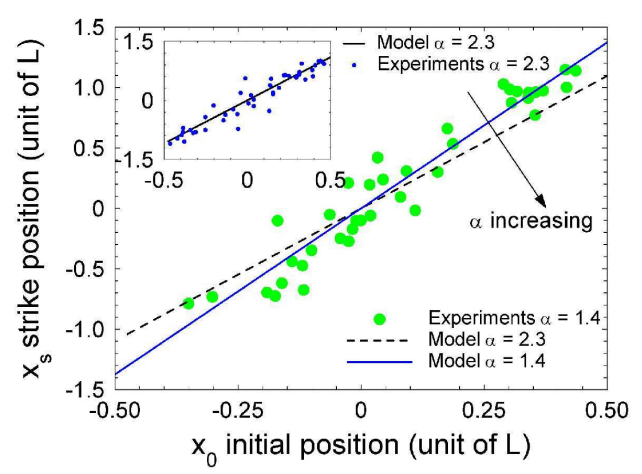

(b)

FiguRE 6. (a) Wall impact speed, $v_{s}$, versus impact position, $x_{s}$, for $a=97 \mu \mathrm{m}$ TiBa glass in pure water, $U=1.2 \mathrm{~m} / \mathrm{s}, \alpha=2.3$. (b) The dependence of the impact position, $x_{s}$, on the initial position, $x_{0}$, of the particle. The solid line indicates the model prediction and the symbols are experimental data; main plot: $a=97 \mu \mathrm{m}$ TiBa glass in pure water, $U=1.2 \mathrm{~m} / \mathrm{s}, \alpha=2.3$. Inset: $a=97 \mu \mathrm{m}$ TiBa glass in 70:30 water:glycerol mixture, $U=1.06 \mathrm{~m} / \mathrm{s}, \alpha=1.4$.

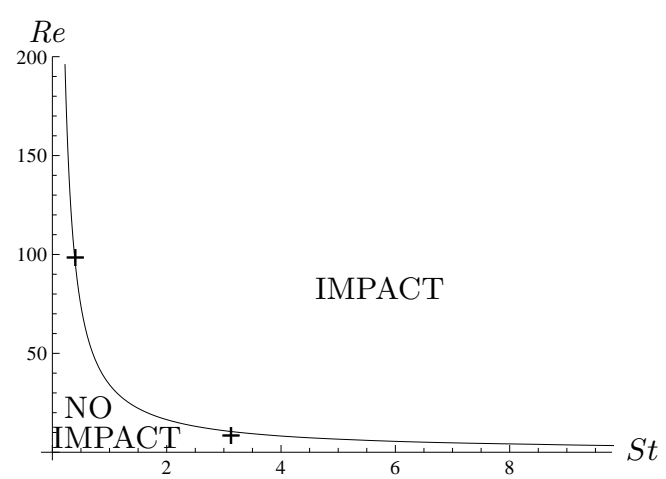

(b)

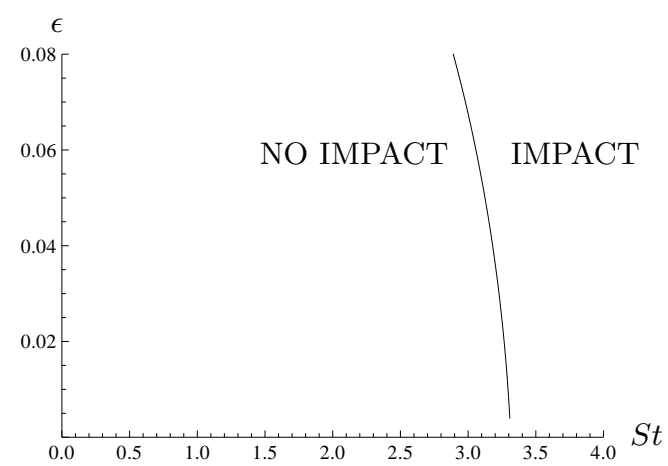

(a)

FiguRE 7. (a) Limiting curve in Re-St space dividing the impact and non-impact zone predicted by the two-dimensional Hiemenz flow field. The data points $(+)$ show the limiting Stokes number for a given Reynolds number predicted by the fully three-dimensional model for simulations with $R e=10$ and $R e=100$; (b) Limiting curve in $\epsilon-S t$ space divides the impact and non-impact zone (where $\epsilon=\alpha / \sqrt{R e}$ and $R e=10$ ).

entrained particles that is not offered by the full three-dimensional simulations or the corresponding experiments.

In figure $7(b)$ the $2 \mathrm{D}$ theory is used to examine the effect of particle size on limit curve prediction, by considering the dependence of $\alpha / \sqrt{R e}$ (which corresponds to the ratio of particle size to T-junction geometry, $\epsilon$ ) on $S t$. This demonstrates that the particle size has only a weak influence on the impact predictions, which is in line with our assumption that the particles do not influence the surrounding flow.

Finally, it only remains to compare the predictions made with the experimental data. The data is analysed via a MATLAB particle-tracing code to identify whether an impact occurs. From this data we define three categories for an individual particle's behaviour: 'impacting', when a particle clearly rebounds off the wall, that is, the rebound velocity away from the wall exceeds zero; 'not impacting', when a particle maintains a distance 
from the wall without collision; and 'barely impacting', when particles just graze the surface of the T-shaped channel and then proceed to the outlets. These classifications are then used to categorize a complete system configuration as 'impacting' if the number of particles that are observed to rebound exceeds $25 \%$ of the total, 'not impacting' if this ratio is less than $1 \%$, and 'barely impacting' for the region in between. The percentages chosen to separate these three classifications were empirically determined, and the reliability of the method was verified to be appropriate via visual inspection of each movie, as well as being used to discriminate those particles that are barely impacting.

We use the observation of relative weak dependence of system behaviour upon particle size made in figure $7(b)$ to express $R e$ in terms of $\alpha$ via the relation $\alpha=\epsilon \sqrt{R e}$, holding $\epsilon$ fixed. This allows the entire parametric dependence of the system to be collapsed onto a single phase plane for $\alpha$ versus St, using the two-dimensional flow eqations (3.2) and (4.12), where the parameter $\alpha$ captures the effect of the viscous boundary layer and the flow regime. The resulting limiting curve predicted by the $2 \mathrm{D}$ particle-tracing model is compared with the full set of experimental data in figure 8 with $\epsilon=0.01$. The agreement between the 2D-model prediction and experimental results is extremely good, with any discrepancies most likely to be arising as a result of a combination of the three-dimensionality of the flow, which is reflected in the more complex 3D numerical simulations, the level of ambiguity in classification of a strike from the experimental data and the fact that some experiments may be taken slightly out of the $z=0$ mid-plane. We thus conclude that the reduced 2D viscous-flow model accurately captures the key features of the T-junction problem for particles that are most likely to impact on the channel surface, which lie within the $z=0$ symmetry mid-plane and are not close to the side walls bounding the flow in the $z$-direction, in a simple manner. These results thus suggest that this reduced $2 \mathrm{D}$ analysis provides insight and a route to quick-scenario testing to determine whether or not an impact will occur for a range of applications. The results are particularly important in scenarios in which time constraints prohibit more lengthy full three-dimensional computational simulations, such as in situations where flow conditions are continuously being altered.

\section{Closing remarks}

The impact of particles entrained in a fluid with the wall in a T-shaped channel have been considered. A series of experiments were conducted for a broad range of system and particle configurations to investigate the effect of the presence of the viscous boundary layer on the resulting impacts on the channel.

Three-dimensional numerical simulations described the flow field, and particle-tracing models enabled the tracking of entrained particles within the flow to determine those which will impact on the channel surface. By exploiting the system symmetry a simplified 2D extensional-flow model was proposed. Comparison of this model with full 3D simulations showed that the model characterizes the typical behaviour well and provided a tractable way of predicting the possibility of a collision for any given system configuration. The 2D model was further validated by comparison with simple experimental observations and shown to agree well. The theory elucidated three key parameters on which the particle collisions depend: the Reynolds number, which characterizes the flow field; the Stokes number, which describes the particle characteristics, and a parameter that relates the particle size to the depth of the viscous boundary layer. The $2 \mathrm{D}$ model provided a route for determining a continuous limiting curve that separates the phase space in which a particle and flow configuration would lead to an impact and those for which an impact would not occur. The $2 \mathrm{D}$ model thus provides a simple but accurate 


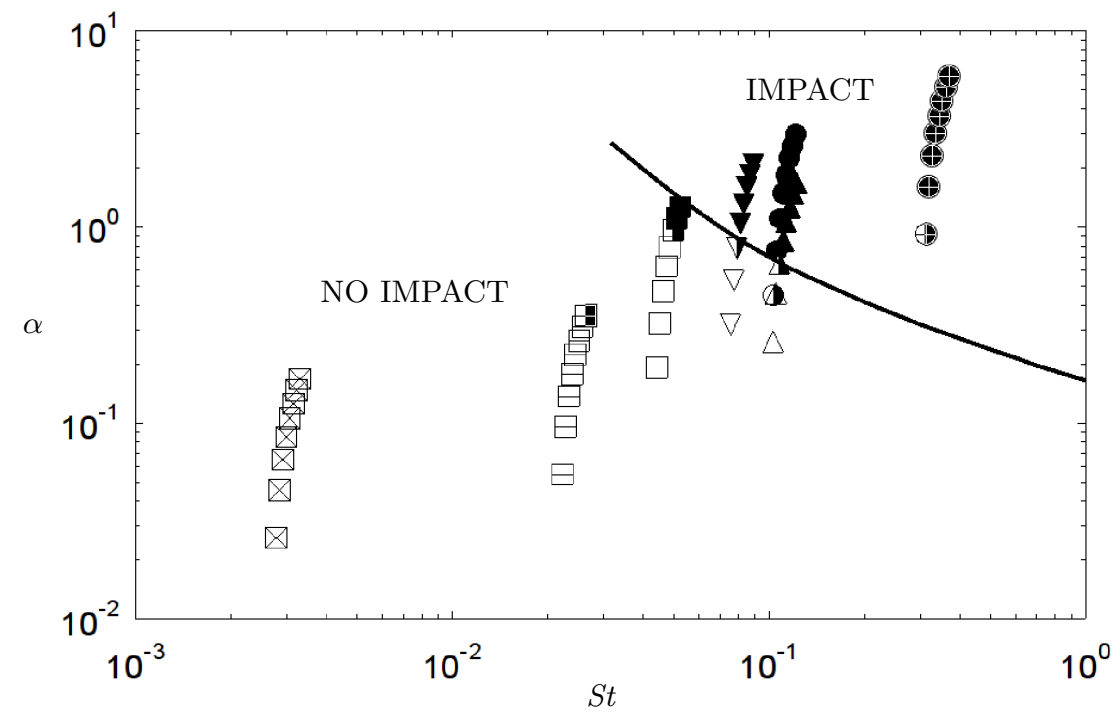

Figure 8. Phase plane $\alpha$ versus St. All the experiments are divided into three main categories: particles 'impacting' (closed symbols), particle 'not impacting' (open symbols) and particle 'barely impacting' (half-filled symbols). The symbols identify the kind of particles used: $a=10 \mu \mathrm{m}$ PMMA ( $\otimes), a=21 \mu \mathrm{m}$ TiBa $(\boxminus), a=75 \mu \mathrm{m} \mathrm{SiO}_{2}(\square), a=97 \mu \mathrm{m}$ TiBa glass $(\triangle)$, $a=128 \mu \mathrm{m} \mathrm{SiO} \mathrm{S}_{2}(\nabla), a=175 \mu \mathrm{m} \mathrm{SiO}_{2}(\mathrm{O})$ and $a=350 \mu \mathrm{m} \mathrm{ZrO}_{2} / \mathrm{SiO}_{2}(\oplus)$. For each system investigated the figure shows seven experimental results obtained for water:glycerol ratios of: 100:0, 90:10, 80:20, 70:30, 60:40, 50:50, 40:60 and 30:70. The solid line represent the 2D model limiting curve that divides the phase plane into 'impact zone' and 'non-impact' zones. The theoretical curve is determined by fixing $\epsilon=0.01$ and varying $R e$ through $R e=(\alpha / \epsilon)^{2}$.

characterization of the behaviour of entrained particles that are most likely to impact on a channel wall within a T-junction flow.

The result of our work provides an easy tool to predict whether or not a particle will produce an impact, and can be incorporated with a specific erosion model to determine the rate at which damage occurs. This study may be generalized to a range of situations involving a dispersion of particles in a constrained geometry.

The ability to assess the possible damage induced by solid particle erosion, especially in a sensitive environment such as the cooling pipe system of a nuclear power plant, where typically the coolant of the system cannot be replaced often, is crucial (Crockett et al. 2010). Determining if a flow field may be potentially dangerous in the presence of a dispersion of particles by simply evaluating a particular combination of dimensionless numbers represents a significant advantage over more complex computational fluid dynamics simulations. In particular, the reduced 2D model presented here provides a limiting curve that immediately identifies the region of potential danger. Thus, such models provide a route to quick-scenario testing that is not offered by 3D simulations, which require long computational time and are discrete, providing only information for a single, specific condition. Knowing the complete impact phase plane greatly simplifies the task of risk evaluation in scenarios where changes in the operative conditions render long computations impractical.

The authors gratefully acknowledge helpful discussions with A. M. Lock, M. Taroni 
and D. Vella, and thank the Princeton Plasma Physics Laboratory (PPPL), in particular P. Heitzenroeder, M. Kalish, C. Neumeyer and M. Mardenfeld for inspiration and conversation. IMG thanks Award No. KUK-C1-013-04, made by King Abdullah University of Science and Technology (KAUST) for support.

\section{REFERENCES}

Adeosun, J. T. \& LAWAL, A. 2009 Numerical and experimental studies of mixing characteristics in a T-junction microchannel using residence-time distribution. Chem. Eng. Sci. 64, 24222432.

Benchaita, M. T., Griffith, P. \& Rabinowicz, E. 1983 Erosion of metallic plate by solid particles entrained in a liquid jet. J. Eng. Ind.(Trans. ASME) 105, 215-222.

Blanchard, D. J., Griffith, P. \& Rabinowicz, E. 1984 Erosion of a pipe bend by solid particles entrained in water. J. Eng. Ind. (Trans. ASME) 106, 213-221.

Bruecker, C. 1997 Study of the three-dimensional flow in a T-junction using a dual-scanning method for three-dimensional scanning-particle-image velocimetry (3-d SPIV). Exp. Thermal and Fluid Sci. 14, 35-44.

Chen, X., McLaury, B. S. \& ShiRazi, S. A. 2006 Numerical and experimental investigation of the relative erosion severity between plugged tees and elbows in dilute gas/solid two-phase flow. Wear 261, 715-729.

Clark, H. M. I. 1991 On the impact rate and impact energy of particles in a slurry pot erosion tester. Wear 147, 165-183.

Clift, R., Grace, J. R. \& Weber, M. E. 1978 Bubbles, drops, and particles. Academic press, New York.

Crockett, H. M., Horowitz, J. S. et al. 2010 Erosion in nuclear piping systems. J. Pressure Vessel Technology 132, 024501.

Dreher, S., Kockmann, N. \& Woias, P. 2009 Characterization of laminar transient flow regimes and mixing in T-shaped micromixers. Heat Transfer Engineering 30 (1-2), 91-100.

Goldman, A. J., Cox, R. G. \& Brenner, H. 1967 Slow viscous motion of a sphere parallel to a plane wall-i motion through a quiescent fluid. Chem. Eng. Sci. 22, 637-651.

Haller, D., Woias, P. \& Kockmann, N. 2009 Simulation and experimental investigation of pressure loss and heat transfer in microchannel networks containing bends and T-junctions. International Journal of Heat and Mass Transfer 52 (11), 2678-2689.

VAN HAREN, SW 2011 Testing DNS capability of OpenFOAM and STAR-CCM. Master's thesis, Delft University of Technology.

Hiemenz, K. 1911 Die grenzschicht an einem in den gleichförmigen flüssigkeitsstrom eingetauchten geraden kreiszylinder (the boundary layer on a circular cylinder in uniform flow). Dingl. Polytec. J. 326, 321-328.

Homann, F. 1936 Der einfluss grosser zähigkeit bei der strömung um den zylinder und um die kugel. ZAMM-Journal of Applied Mathematics and Mechanics/Zeitschrift für Angewandte Mathematik und Mechanik 16, 153-164.

JASAK, H. 1996 Error analysis and estimation for the finite volume method with applications to fluid flows. PhD thesis, University of London and Imperial College.

Kockmann, N. \& Roberge, D. M. 2011 Transitional flow and related transport phenomena in curved microchannels. Heat Transfer Engineering 32, 595-608.

Levy, A. V. 1995 Solid Particle Erosion and Erosion-corrosion of Materials. ASM Intl.

LeVy, A. V., JeE, N. \& YAU, P. 1987 Erosion of steels in coal-solvent slurries. Wear 117, $115-127$.

Li, X., Hunt, M.L. \& Colonius, T. 2012 A contact model for normal immersed collisions between a particle and a wall. J. Fluid Mech. 1, 1-23.

Lynn, R. S., Wong, K. K. \& Clark, H. M. I. 1991 On the particle size effect in slurry erosion. Wear 149, 55-71.

Mclaury, B. S., Wang, J., Shirazi, S. A., Shadley, J. R. \& Rybicki, E. F. 1997 Solid particle erosion in long radius elbows and straight pipes. In SPE Annual Technical Conference and Exhibition.

Pasol, L., Martin, M., Ekiel-Jezewska, M. L., Wajnryb, E., Blawzdziewicz, J. \& 
Feuillebois, F. 2011 Motion of a sphere parallel to plane walls in a Poiseuille flow. Application to field-flow fractionation and hydrodynamic chromatography. Chem. Eng. Sci. 66, 4078-4089.

Richardson, J. F., Coulson, J. M., Harker, J. H. \& Backhurst, J. R. 2002 Chemical Engineering: Particle Technology and Separation Processes. Butterworth-Heinemann.

Rubinow, S. I. \& Keller, J. B. 1961 The transverse force on a spinning sphere moving in a viscous fluid. J. Fluid Mech. 11, 447-459.

Shivamurthy, R. C., Kamaraj, M., Nagarajan, R., Shariff, S. M. \& Padmanabham, G. 2010 Slurry erosion characteristics and erosive wear mechanisms of co-based and ni-based coatings formed by laser surface alloying. Metall. Mat. Trans. A 41, 470-486.

Sommerfeld, M. \& Huber, N. 1999 Experimental analysis and modelling of particle-wall collisions. Int. J. Multiphase Flow 25, 1457-1489.

Tu, J. Y. 2000 Numerical investigation of particulate flow behavior in particle-wall impaction. Aerosol Sci. \& Tech. 32, 509-526.

Tu, J. Y., Yeoh, G. H., Morsi, Y. S. \& YAnG, W. 2004 A study of particle rebounding characteristics of a gas-particle flow over a curved wall surface. Aerosol Sci. \& Tech. 38, 739-755.

Wong, S. H., Ward, M. C. L. \& Wharton, C. W. 2004 Micro T-mixer as a rapid mixing micromixer. Sensors and Actuators B: Chemical 100, 359-379.

Wood, R. J. K. \& Jones, T. F. 2003 Investigations of sand-water induced erosive wear of AISI 304L stainless steel pipes by pilot-scale and laboratory-scale testing. Wear 255, 206-218.

YANG, F. L. \& Hunt, M. L. 2008 A mixed contact model for an immersed collision between two solid surfaces. Phil. Trans. Roy. Soc. A 366, 2205-2218.

Zhang, Y., Reuterfors, E. P., McLaury, B. S., Shirazi, S. A. \& Rybicki, E. F 2007 Comparison of computed and measured particle velocities and erosion in water and air flows. Wear 263, 330-338.

Zhaо, H. X., Yамамото, M. \& Matsumura, M. 1995 Slurry erosion properties of ceramic coatings and functionally gradient materials. Wear 186, 473-479.

\section{Appendix A. Three-dimensional numerical simulations}

We performed computational studies of single-phase flow in a T-junction using a finitevolume Navier-Stokes (NS) solver on unstructured meshes. Such NS-solvers are a standard tool, e.g., for the analysis of the flow in microreactors (Kockmann \& Roberge 2011). We used the open-source package OpenFOAM (Jasak 1996), which is widely used in industry and academia, and has demonstrated its parallel performance on various large-scale computer clusters. OpenFOAM can be seen as a mature tool for direct numerical simulations (DNS) of fluid flows in complex geometries, e.g. it has been applied recently to study turbulent channel flows (van Haren 2011). As DNS aims to resolve all details of the flow, it requires an extremely high spatial and temporal resolution during the numerical simulation. Specifically, we tested various computational grids for the flow at $R e=600$, with cell counts between $375 \mathrm{k}$ and 2 Mio. cells, with refined grids near the walls to capture the large gradients of the streamwise velocity component especially near the bottom wall. Also, a small enough time step was used to ensure a Courant number of $C o<0.3$ in order to accurately capture the transients of the flow. These settings lead to typical simulation times of $\mathrm{O}(10)$ days for $\mathrm{O}(100)$ dimensionless time units that are needed to obtain a sufficiently large data set to characterize unsteady flow features (steady flow was observed up to $R e \approx 540$ ). All simulations were performed in parallel on a Quad-Core CPU cluster. Based on our results for various grid spacings and numerical settings, we are confident that our DNS results for flows in T-junctions up to $R e=600$ are independent of numerical resolution effects within an error tolerance of approximately $5 \%$. DNS results reported for higher Reynolds numbers are connected to 
somewhat larger inaccuracies, since the boundary layer thickness becomes smaller, and discretization errors increase.

Our DNS simulations were used (i) to obtain a qualitative understanding of the flow field (an example is shown in figure $2(a)$ ) and (ii) to extract data (instantaneous and time-averaged pressure and velocity distributions) for each point in the 3D computational domain. The latter was realized by extracting the results in two-dimensional slices, and subsequently re-sampling the data on a regular grid in MATLAB; sampling was performed using MATLAB's 'griddata' functionality using spline interpolation. The data was then imported into Mathematica ${ }^{\circledR}$ to track particles dispersed in the flow as described in $\S 4$. 



\section{RECENT REPORTS}

12/66 Morphoelastic Rods Part I: A Single Growing Elastic Rod

Moulton

Lessinnes

Goriely

12/67 Wrinkling in the deflation of elastic bubbles

Aumaitre

Knoche

Cicuta

Vella

12/68 Indentation of ellipsoidal and cylindrical elastic shells

Vella

Ajdari

Vaziri

Boudaoud

12/69 Memory of Recessions

Cross

McNamara

Pokrovskiif

12/70 An estimate of energy dissipation due to soil-moisture hysteresis

McNamara

12/71 The Mathematics Behind Sherlock Holmes: A Game of Shadows

Goriely

Moulton

12/72 Some observations on weighted GMRES

Güttel

Pestana

12/73 Bounds on the solution of a Cauchy-type problem involving a Furati weighted sequential fractional derivative

12/74 Static and dynamic stability results for a class of threedimensional configurations of Kirchhoff elastic rods

Majumdar

Goriely

12/75 Error estimation and adaptivity for incompressible, nonlinear (hyper)elasticity

Whiteley

Tavener

12/76 A note on heat and mass transfer from a sphere in Stokes flow at low Péclet number

Bell

Byrne

Whiteley

Waters

12/77 Effect of disjoining pressure in a thin film equation with non- Moulton uniform forcing

Lega

12/78 A Review of Mathematical Models for the Formation of Vascular Networks

Scianna

Bell

Preziosi

12/79 Fast and Accurate Computation of Gauss-Legendre and Gauss-

Hale Jacobi Quadrature Nodes and Weights

Townsend

$12 / 80$ On the spectral distribution of kernel matrices related to radial basis functions

Wathen

Zhu 
12/81 Inner product computation for sparse iterative solvers on dis- Zhu tributed supercomputer

$\mathrm{Gu}$

Liu

12/82 A new pathway for the re-equilibration of micellar surfactant solutions

Griffiths

Breward

Colegate

Dellar

Howell

Bain

12/83 Object-Oriented Paradigms for Modelling Vascular Tumour Growth: a Case Study

Connor

Cooper

Byrne

Maini

McKeever

12/84 Chaste: an open source $\mathrm{C}++$ library for computational physiology and biology

Mirams

Arthurs

Bernabeu

Bordas

Cooper

Corrias

Davit

Dunn

Fletcher

Harvey

Marsh

Osborne

Pathmanathan

Pitt-Francis

Southern

Zemzemi

Gavaghan

12/85 A two-pressure model for slightly compressible single phase flow

Schlackow in bi-structured porous media

Marguerat

Proudfoot

Bähler

Erban

Gullerova

12/86 Boolean modelling reveals new regulatory connections between

Lovrics transcription factors orchestrating the development of the ventral spinal cord

Gao

Juhász

Bock

Byrne

Dinnyés

Kovács

12/87 Asymptotic solutions of glass temperature profiles during steady

Taroni optical fibre drawing

Breward

Cummings

Griffiths

Morgan

Breward 
Copies of these, and any other OCCAM reports can be obtained from:

Oxford Centre for Collaborative Applied Mathematics Mathematical Institute

24 - 29 St Giles'

Oxford

OX1 3LB

England

www.maths.ox.ac.uk/occam 